\title{
Ueber das Zöllner'sche Photometer.
}

Die folgenden Zeilen sollen die Beobachter, welche das Zöllnersche Photometer benutzen, auf eine kleine $\mathrm{Ab}$ änderung dieses Instrumentes aufmerksam machen, welche, wie ich hoffe, bei der Messung heller Sterne von Vortheil sein wird.

Der Oculartheil des Photometers besteht aus zwei gegeneinander senkrechten Röhren. Die Axe der einen fällt mit der Axe des Fernrohrs zusammen, die Axe der zweiten steht senkrecht darauf. In dem einen Theil des letztern Rohres sind die Nicols angebracht; den andern Theil derselben habe ich ausgehöhlt und so eingerichtet, dass das Photometer-Ocular hineingeschoben werden kann.

Das Photometer hat nun demnach zwei Oculare: das gewöhnliche und ein seitliches.

Die in das gewöhnliche Ocular gelangenden Strahlen des natürlichen Sterns haben die Spiegelglasplatte passirt, diejenigen des künstlichen Sterns sind von derselben reflectirt. Beim Seitenocular haben umgekehrt dic Strahlen des künstlichen Sterns directen Zugang und die des natür-

Moskau I 884 August I 3/25. lichen sind reflectirt. Im ersten Falle sehen wir einen natürlichen und zwei künstliche Sterne, im letzten einen kunstlichen und zwei Bilder des naturlichen.

Angenommen, dass für einen bestimmten Stern lie Ablesung des Intensitätskreises für das gewöhnliche Ocviłr $\varphi$, für das Seitenocular $\varphi^{\prime}$ ist und $\log \sin ^{2} \varphi=L$, so wird $\log \sin ^{2} \varphi^{\prime}$ nahezu gleich $L-1.64$.

Die Quantität r:64 entspricht einer Grössenändeiung um 4.7 Grössenclassen. Wenn sich also mit dem gewöhnlichen Ocular Sterne von der Grösse $n, n^{\prime} \ldots n^{n}$ beobachten lassen, so wird man mit dem Seitenocular ebenso beque $m$ und ebenso genau Sterne von der Grösse $(n-4.7)$, $\left(n^{\prime}-4.7\right) \ldots,\left(n^{n}-4.7\right)$ beobachten könnnen.

Zur Abschwächung der natürlichen Sterne kann man auch die Spiegelglasplatte durch eine Platte mehr oder weniger dunklen Neutralglases ersetzen. Dabei bleibt der eine der künstlichen Sterne ungeändert, während der andere zugleich mit dem natürlichen abgeschwächt wird.

\section{Entdeckung eines nenen Planeten (244) auf der Sternwarte in Wien durch Dr. J. Palisa.}

Die Centralstelle erhielt folgendes Telegramm, welches sofort weiter befördert wurde:

Planet Palisa 14. Octuber ${ }_{10} 0^{\mathrm{h}} 4^{\mathrm{m}} . \mathrm{I}$ M. Z. Wien AR. $=34^{\circ} 36^{\prime} 35^{\prime \prime}, \quad \mathrm{PD} .=76^{\circ} 12^{\prime} 53^{\prime \prime}$

Bewegung in AR. - $14^{\prime}$ und in PD. $+6^{\prime}$.

Weiss.

\section{Beobachtungen des Cometen 1884 Wolf.}

A m Refractor der Kieler Sternwarte.

\begin{tabular}{|c|c|c|c|c|c|c|c|c|c|}
\hline I 884 & M. Z. Kiel & $\Delta c$ & $\Delta d$ & Vgl. & $\alpha$ app. & $\log p \cdot A$ & $\delta$ app. & $\log p .4$ & $*$ \\
\hline Oct. 10 & $9^{h} 54^{\mathrm{m} I 1} 45^{\mathrm{s}}$ & $-\mathrm{I}^{\mathrm{II}} 26.52$ & + r' 5.0 & I I & $21^{\mathrm{h}} 32^{\mathrm{m}} 39^{\mathrm{s}} \cdot 34$ & 9.18 I & + I $2^{\circ} 54^{\prime} 43^{\prime \prime} 6$ & 0.773 & 1 \\
\hline I 3 & $9 \circ 39$ & +2 I $2.8 \mathrm{I}$ & - 633.6 & 10 & $\begin{array}{lll}21 & 3^{6} & 43.88\end{array}$ & 8.922 & +1 I 2735.2 & 0.779 & 2 \\
\hline
\end{tabular}

Mittlere Oerter der Vergleichsterne für ${ }_{1} 884.0$.

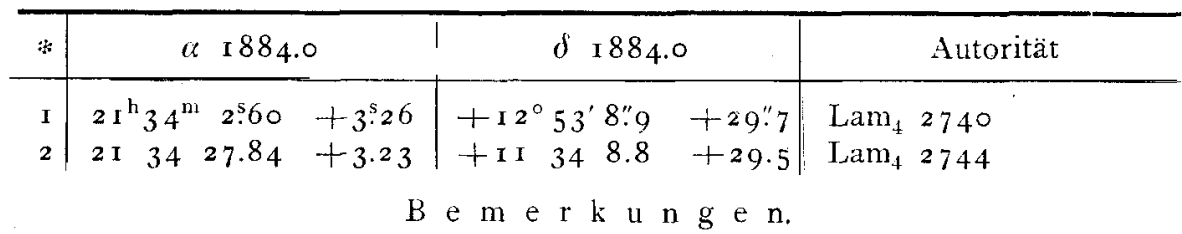

Oct. 10. Mikrometer mit dicken Metallfäden bei fast ganz dunklem Felde. Luft ziemlich gut.

Oct. I 3. Helle Fäden. Mehrfach Unterbrechung durch Wolken.

Correction der Ephemeride von Prof. Krueger in A. N. 2619 : Oct. Io + $2^{\mathrm{s}}-0.2$, Oct. 13 + $1^{\mathrm{s}}-0.2$.

Vergleichstern 2 ist DM. $+\mathrm{I}^{\circ} 4566 \mathrm{~K}$. Die AR. desselben nach der Königsberger Zone 28 ist um $6^{\mathrm{s}} \mathrm{kleiner}$ als Lam $_{4} \mathbf{2 7 4 4}$, wie Lamont auch selbst schon angiebt. Die Lamont'sche Position habe ich durch Vergleichung mit 2 anderen Sternen bestätigt gefunden.

Kiel I 884 Oct. I 4 . 